\title{
Determinação das constantes cinéticas de degradação do ácido ascórbico em purê de pêssego: efeito da temperatura e concentração
}

\author{
Determination of reaction rate constants for ascorbic acid degradation \\ in peach pureé: effect of temperature and concentration
}

\author{
Ricardo Peraça TORALLES ${ }^{1}$, João Luiz VENDRUSCOLO ${ }^{2 *}$, Claire Tondo VENDRUSCOLO ${ }^{3}$, \\ Francisco Augusto Burkert DEL PINO ${ }^{4}$, Pedro Luiz ANTUNES
}

\begin{abstract}
Resumo
O ácido ascórbico, vitamina C, é usado extensivamente na indústria de alimentos, não só devido ao seu valor nutricional, mas devido a suas contribuições funcionais na qualidade do produto. Existem muitos estudos sobre a estabilidade cinética do ácido ascórbico em bebidas, mas nenhum estudo foi encontrado sobre as constantes cinéticas de degradação do ácido ascórbico adicionado em purê de pêssego. Neste trabalho, estudou-se a cinética de degradação do ácido ascórbico em purê de pêssego da cultivar Jade, em condições anaeróbicas e na faixa de 70 a $90^{\circ} \mathrm{C}$. As concentrações de purês testadas foram 12, 22 e $32^{\circ}$ Brix. A análise cinética dos dados sugere que a degradação foi significativamente representada pelos modelos cinéticos de zero e primeira ordem. A velocidade de degradação do ácido ascórbico foi dependente da temperatura. A energia de ativação média foi de $45 \mathrm{~kJ} \cdot \mathrm{mol}^{-1} \mathrm{e}$ independente da concentração de sólidos solúveis.
\end{abstract}

Palavras-chave: ácido ascórbico; purê de pêssego; cinética da degradação.

\begin{abstract}
Ascorbic acid (vitamin C) is extensively used in the food industry, not only for its nutritional value, but also for its many functional contributions to product quality. There have been many studies on the stability of ascorbic acid in different beverages, but no study was found on the reaction rate constants for ascorbic acid degradation in peach purée. In this work, the degradation of ascorbic acid in Jade peach purée was studied in anaerobic conditions and from $70-90{ }^{\circ} \mathrm{C}$. The peach purée concentrations tested were 12,22 and $32{ }^{\circ} \mathrm{Brix}$. The kinetic analysis of the data suggests that the degradation was significantly represented by zero and first-order kinetic models. The rate of ascorbic acid degradation in peach purée was temperature dependent. The average activation energy was $45 \mathrm{~kJ}^{-m o l^{-1}}$ and independent of the concentration of soluble solids.
\end{abstract}

Keywords: ascorbic acid; peach purée; degradation kinetics.

\section{Introdução}

Purê de pêssego assepticamente processado ou congelado é uma "commodity" do mercado mundial sendo usualmente comercializada a $32^{\circ} \mathrm{Brix}$, mas também é comercializada em concentrações mais baixas. Purês são utilizados na elaboração de sucos e néctares de frutas e, tradicionalmente, são elaborados pela combinação de tratamento térmico e de inibidores. Entretanto, esses produtos sofrem alterações durante o processamento térmico e armazenamento, que podem ser significativas, afetando cor, sabor, aroma e valor nutricional (BUGLIONE; LOZANO, 2002; GARZA et al., 1999; LOZANO; IBARZ, 1997; LUH, 1980). O escurecimento não enzimático é considerado uma das maiores causas de perda de qualidade durante o processamento e armazenamento dessas "commodities"(BABSKY; TORIBIO; LOZANO, 1986; BURDURLU; KARADENIZ, 2003; KOCA;
SELEN BURDURLU; KARADENIZ, 2003). Estudos nessa direção incluem a degradação do ácido ascórbico (JOHNSON, 1995), a reação de Maillard e a pirólise dos carboidratos (BUERA et al., 1987; HOSNEY, 1984; PETRIELLA, 1985).

A adição do ácido ascórbico é comum na indústria de processamento de frutas, especialmente em sucos e purês (PORRETTA, 1991; SMOOT, NAGY, 1980). O ácido ascórbico não só pode restabelecer valor nutricional perdido durante processamento, como também inibir o escurecimento enzimático resultando em melhoria da cor e da palatabilidade (ASHURST 1995; FREEDMAN; FRANCIS, 1984). As polifenoloxidases (PPO), principais enzimas responsáveis pelo escurecimento enzimático em frutas, catalisam a oxidação dos

Recebido para publicação em 28/3/2006

Aceito para publicaçào em 17/12/2007 (001713)

${ }^{1}$ Departamento de Química, Centro Federal de Tecnologia, Pelotas - RS, Brasil, E-mail: torashow@cefetrs.tche.br

${ }^{2}$ Clima Temperado, Empresa Brasileira de Pesquisa Agropecuária - EMBRAPA, Km 78, BR 392, CP 403, CEP 960001-970, Pelotas - RS, Brasil,

E-mail:vendrusc@cpact.embrapa.br

3 Centro de Biotecnologia, Universidade Federal de Pelotas - UFPel, Pelotas - RS, Brasil, E-mail: claire@ufpel.tche.br

${ }^{4}$ Departamento de Bioquímica, Universidade Federal de Pelotas - UFPel, Pelotas - RS, Brasil, E-mail: edelpino@ufpel.tche.br

${ }_{5}^{5}$ Departamento de Ciência e Tecnologia de Alimentos, Universidade Federal de Pelotas - UFPel, Pelotas - RS, Brasil, E-mail: pantunes@ufpel.tche.br

${ }^{*}$ A quem a correspondência deve ser enviada 
fenóis naturalmente encontrados nas frutas formando quinonas (GIRNER et al., 2002; OKTAY et al., 1995; TORALLES et al., 2004). Estas, por sua vez, podem polimerizar formando melanoidinas que causam a pigmentação marrom das frutas e seus derivados (VÁMOS-VIGYÁZÓ, 1981). O ácido ascórbico reverte o escurecimento por redução da quinona a fenol. É também um potente antioxidante devido a sua capacidade de transformar radicais livres de oxigênio em formas inertes, que é acompanhada da conversão do ácido ascórbico em deidroascórbico, sua forma oxidada (Figura 1).

Apesar de todos os benefícios citados, os estudos relativos à degradação do ácido ascórbico durante o processamento ou armazenamento são conhecidos para um pequeno universo de sucos de frutas, incluindo concentrado de laranja (JOHNSON; BRADDOCK; CHEN, 1995), suco de limão (ROBERTSON; SAMANIEGO, 1986), suco de uva (LEE; NAGY, 1988; SMOOT; NAGY, 1980), suco de romã e néctar de cereja (OZKAN, 2004). Tanto uma cinética de ordem zero como uma de primeira ordem têm sido sugeridas para explicar a degradação do ácido ascórbico, existindo ampla variação na magnitude das constantes de velocidades devido a inúmeros fatores que podem influenciar na velocidade de degradação, tais como: a temperatura, a concentração de sal e açúcar, o pH, o oxigênio, as enzimas, os catalisadores metálicos, a concentração inicial de ácido e a relação inicial de ácido ascórbico-deidroascórbico (FENNEMA, 1993; JOHNSON; BRADDOCK; CHEN, 1995; OZKAN; KIRCA; CEMEROGLU, 2004). Além disso, novos fatores vêm sendo descobertos, por exemplo, a remoção de aminoácidos em suco concentrado de kiwi que foi efetiva para controlar a perda do ácido ascórbico, diferente da remoção de compostos fenólicos (WONG; STANTON, 1993). Também, sabe-se que o mecanismo de degradação do ácido ascórbico pode ocorrer via aeróbica ou anaeróbica (FENNEMA, 1993; ROBERTSON; SAMANIEGO, 1986).

No processamento térmico de purê de pêssego é recomendada a utilização de ácido ascórbico para prevenir reações de escurecimento. A adição de ácido ascórbico é feita logo após o refino da polpa e antes da pasteurização (LUH, 1980). Toralles et al. (2004), estudando o efeito combinado do $\mathrm{pH}$, da temperatura e do ácido ascórbico na atividade da PPO, observaram várias condições ótimas para inibir o escurecimento enzimático provocado pela $\mathrm{PPO}$ de pêssego Granada. $\mathrm{O}$ escurecimento não enzimático durante o processamento de purê de pêssego foi recentemente estudado por Garza et al. (1999). Estes acompanharam a cinética de formação de compostos coloridos por espectrofotometria a $420 \mathrm{~nm}$, bem como a cinética de for-
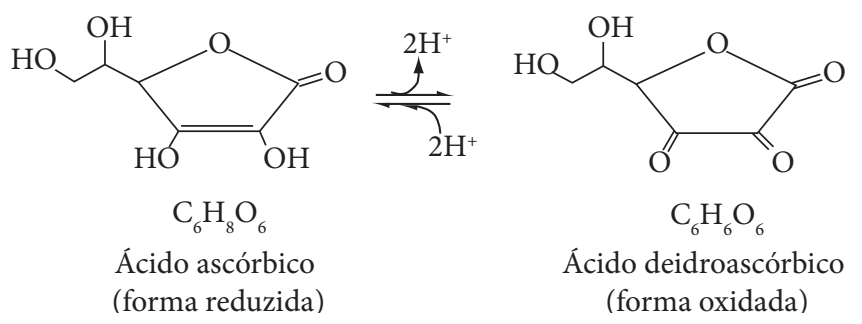

Figura 1. Oxidação do ácido ascórbico em deidroascórbico. mação do hidroxidometilfurfural (HMF) a partir de hexoses. Entretanto, na literatura pertinente, não é reportado estudo sobre a degradação do ácido ascórbico em purê de pêssego durante o processamento.

O objetivo deste trabalho foi determinar as constantes cinéticas de degradação do ácido ascórbico em temperaturas normalmente usadas durante o branqueamento, a pasteurização e a concentração de purê de pêssego.

\section{Material e métodos}

\subsection{Frutas}

Frutas de pêssego maduro e de boa aparência da cultivar Jade, de baixo potencial de escurecimento, safra 2002/2003, foram colhidas em pomar comercial na região de Pelotas/RS e armazenadas em câmara fria entre 1 e $3^{\circ} \mathrm{C}$.

\subsection{Preparo do purê}

O processamento do purê de pêssego concentrado a $32{ }^{\circ}$ Brix ocorreu na planta piloto da Universidade Federal de Pelotas-CAVG. As frutas foram selecionadas, lavadas, descaroçadas e descascadas, utilizando o mesmo procedimento para o processamento de pêssegos em calda. Para facilitar o processo de formação de purê e inativar as enzimas responsáveis pelo escurecimento, as frutas foram branqueadas em termoinativador enzimático tubular contínuo, com controle da temperatura da jaqueta $(\mathrm{Tj})$, da temperatura de referência da polpa (Tp) e do tempo de branqueamento $(\mathrm{t})$. As condições da operação de branqueamento foram: $\mathrm{Tj}=95^{\circ} \mathrm{C} ; \mathrm{Tp}=75^{\circ} \mathrm{C}$ e $\mathrm{t}=4$ minutos. Logo após, passaram por uma primeira despolpadeira ("finisher") com tela inox de $1 \mathrm{~mm}$ de abertura, seguida de uma segunda com abertura de $0,5 \mathrm{~mm}$. Posteriormente, foi adicionado ácido ascórbico ( $0,08 \%$ em peso), centrifugado continuamente em um separador Westfallen, modelo KO 2006 a 8380 rpm e em seguida homogeneização em homogeneizador marca Kirchfeld, modelo HL a $10.000 \mathrm{kPa}$. Finalmente, o purê foi concentrado sob vácuo até $32^{\circ} \mathrm{Brix}$. O equipamento utilizado foi um evaporador marca Wiegand de filme descendente, com circulação forçada e com a capacidade de remover $30 \mathrm{~kg} . \mathrm{H}_{2} \mathrm{O}_{\mathrm{h}}{ }^{-1}$. As amostras de purê foram fechadas em latas, seguido de tratamento térmico atmosférico e congeladas a $-20^{\circ} \mathrm{C}$.

\subsection{Preparo das amostras}

Purê concentrado de pêssego a $32^{\circ}$ Brix foi diluído para 22 e $12{ }^{\circ}$ Brix com água deionizada. Quantidades conhecidas das amostras foram colocadas em tubos de $10 \mathrm{~mL}$ com tampas. No espaço vazio de cada tubo foi passada uma corrente de $\mathrm{N}_{2}$ para criar condições anaeróbicas. Dez tubos, em duplicata, foram preparados para cada combinação de concentração e temperatura.

\subsection{Tratamento térmico}

Os tubos foram colocados em um banho com circulação de água (Marconi, Piracicaba - SP, Brasil) para aquecimento. As amostras foram aquecidas entre 70 e $90{ }^{\circ} \mathrm{C}$ para diferentes 
períodos de tempos. A temperatura foi monitorada com termômetro digital. Posteriormente, as amostras foram rapidamente resfriadas e a concentração do ácido ascórbico foi determinada. Os tratamentos foram:

a) Concentrações do purê: 12,22 e $32^{\circ}$ Brix;

b) Temperaturas: $70,75,80,85$ e $90^{\circ} \mathrm{C}$; e

c) Tempos: $0,20,40,60,80,100,120,140,160$ e $180 \mathrm{mi}-$ nutos.

\subsection{Medida do ácido ascórbico}

O teor de ácido ascórbico no purê foi determinado por um método volumétrico usando 2,6-dicloro indofenol, método oficial 967.21 da AOAC (2000).

\subsection{Determinação das constantes cinéticas}

A cinética da degradação térmica do ácido ascórbico foi estudada entre 70 e $90^{\circ} \mathrm{C}$, em condições anaeróbicas, para três concentrações de purê: 12, 22 e $32^{\circ}$ Brix. As constantes cinéticas de zero e de primeira ordem foram calculadas usando os seguintes modelos cinéticos:

$\mathrm{C}_{\mathrm{AO}} \cdot \mathrm{X}_{\mathrm{A}}=\mathrm{kt}$

$\log \left(1-\mathrm{X}_{\mathrm{A}}\right)=-(\mathrm{k} / 2.303) \mathrm{t}$ or $\operatorname{In}\left(1-\mathrm{X}_{\mathrm{A}}\right)=-\mathrm{kt}$

em que $\mathrm{C}_{\mathrm{AO}}$ é a concentração inicial de ácido ascórbico no purê de pêssego, $\mathrm{X}_{\mathrm{A}}$ é a fração convertida de ácido ascórbico após um tempo de aquecimento ' $\mathrm{t}$ ' em minutos e $1-\mathrm{X}_{\mathrm{A}}$ é a fração retida de ácido ascórbico.

\subsection{Efeito da temperatura nos parâmetros cinéticos}

O efeito da temperatura na constante cinética e coeficiente de temperatura $\left(Q_{10}\right)$ foram estimados a partir das constantes cinéticas de primeira ordem calculadas pela Equação 2 e pelas seguintes equações:

$\operatorname{In}(\mathrm{k})=-\mathrm{Ea} / \mathrm{RT}+\operatorname{In} \mathrm{A}$

$\mathrm{Q}_{10}=\frac{\mathrm{k}_{(\mathrm{T}+10)}}{\mathrm{k}_{\mathrm{T}}}$

em que R é a constante universal dos gases (8.314 J.mol-1 $\cdot \mathrm{K}^{-1}$ ), T é a temperatura em Kelvin e A é chamado fator pré-exponencial.

\subsection{Análise estatística}

Os coeficientes de regressão foram estimados por regressão linear usando o software STATISTICA (STATSOFT, 1998). Quando aplicável, os resultados foram analisados por análise de variância (ANOVA) utilizando o mesmo programa.

\section{Resultados e discussão}

\subsection{Cinética de degradação do ácido ascórbico}

As magnitudes das constantes cinéticas das Equações 1 e 2, determinadas por regressão linear, estão na Tabela 1 . Ambos os modelos foram estatisticamente significativos $(\mathrm{p} \leq 0,01)$ para descrever a degradação do ácido ascórbico em todas as condições experimentais estudadas.

As constantes cinéticas de zero e de primeira ordem variaram, respectivamente, de $22,75 \cdot 10^{-4}$ até $172,00 \cdot 10^{-4} \mathrm{mM}^{-1} \cdot \mathrm{min}^{-1}$ e de $4,97 \cdot 10^{-4}$ até $14,3 \cdot 10^{-4} \mathrm{~min}^{-1}$ em purê de pêssego a $12,22 \mathrm{e}$ $32{ }^{\circ}$ Brix, na faixa de temperatura de 70 a $90{ }^{\circ} \mathrm{C}$. Johnson, Braddock e Chen (1995) encontraram valores um pouco menores para as constantes cinéticas de primeira ordem em suco de laranja. A possível explicação para esse fato é que o alto conteúdo de antocianinas em pêssegos pode ter acelerado a degradação do ácido ascórbico. Este tipo de comportamento já foi observado em suco de romã (MARTÍ, 2001).

Na Tabela 2 foi feita uma análise comparativa da magnitude das constantes cinéticas de primeira ordem para reações degradativas que acontecem durante o processamento de purê não concentrado de pêssego a $75^{\circ} \mathrm{C}$. Nesta típica temperatura de referência utilizada durante o branqueamento e a concentração do purê, as constantes cinéticas de degradação do ácido ascórbico e da sacarose foram da mesma magnitude e maiores do que as constantes cinéticas de 'L', 'a' e 'b' para a degradação da cor de purê de pêssego. Por outro lado, foram bem menores do que as constantes de desnaturação da polifenoloxidase (PPO) e peroxidase (POD) de pêssego da cultivar Granada, indicando que o escurecimento enzimático é de rápido controle e as reações de escurecimento não enzimático são muito lentas. A dimensão desse fato cinético é a de que o ácido ascórbico, além de todos os benefícios citados devido a sua adição, contribuiu muito pouco para as mudanças da cor natural da fruta durante o processamento do purê de pêssego.

A Figura 2a representa graficamente a degradação do ácido ascórbico em purê de pêssego a $32^{\circ} \mathrm{Brix}$, segundo uma cinética de ordem zero. A fração convertida $\left(\mathrm{X}_{\mathrm{A}}\right)$, ou simplesmente conversão, é uma variável conveniente utilizada em lugar da concentração de ácido ascórbico e sua conversão aumenta com o incremento da temperatura ao longo do tempo. Na temperatura de $70{ }^{\circ} \mathrm{C}$, a conversão do ácido ascórbico foi de aproximadamente $10 \%$ após 180 minutos, e semelhante perda foi observada durante 80 minutos a $90{ }^{\circ} \mathrm{C}$, sugerindo que o incremento da temperatura afeta profundamente a sua cinética. Por outro lado, a degradação do ácido ascórbico também descreveu uma cinética aparente de primeira ordem, conforme Figura 2b. Esse duplo comportamento cinético também foi observado nos purês a 12 e $22^{\circ} \mathrm{C}$. Recentemente, Ozkan, Kirca e Cemeroglu (2004) observaram que o modelo de ordem zero ajustou-se melhor que o de primeira ordem para degradação do ácido ascórbico em suco de laranja e néctar de cereja durante o armazenamento.

A cinética de degradação do ácido ascórbico também foi dependente da concentração do purê (Figura 3), sendo maior a $32^{\circ}$ Brix do que a 12 e $22^{\circ} \mathrm{Brix}$, mas essa dependência foi baixa. Em sucos concentrados de laranja, a degradação do ácido as- 
Tabela 1. Constante de degradação do ácido ascórbico em três diferentes concentrações de purês de pêssego.

\begin{tabular}{|c|c|c|c|c|c|c|}
\hline \multirow{2}{*}{$\begin{array}{c}\text { Concentração } \\
\left({ }^{\circ} \text { Brix }\right)\end{array}$} & \multirow{2}{*}{$\begin{array}{c}\text { Temperatura } \\
\left({ }^{\circ} \mathrm{C}\right)\end{array}$} & \multirow{2}{*}{$\begin{array}{l}\mathrm{C}_{\mathrm{Ao}} \\
(\mathrm{mM})\end{array}$} & \multicolumn{2}{|c|}{ Ordem zero $^{a}$} & \multicolumn{2}{|c|}{ Primeira ordem $^{\mathrm{a}}$} \\
\hline & & & $\begin{array}{c}\mathrm{k} \times 10^{4} \\
\left(\mathrm{mM}^{-1} \cdot \mathrm{min}^{-1}\right)\end{array}$ & $\mathrm{R}^{2}$ & $\begin{array}{l}\mathrm{k} \times 10^{4} \\
\left(\mathrm{~min}^{-1}\right)\end{array}$ & $\mathrm{R}^{2}$ \\
\hline \multirow[t]{5}{*}{12} & 70 & 4,78 & $22,75 \pm 1,92$ & 0,987 & $4,97 \pm 0,42$ & 0,986 \\
\hline & 75 & 5,91 & $29,96 \pm 1,77$ & 0,993 & $6,10 \pm 0,32$ & 0,994 \\
\hline & 80 & 4,90 & $34,55 \pm 1,34$ & 0,997 & $7,53 \pm 0,32$ & 0,996 \\
\hline & 85 & 5,02 & $44,13 \pm 2,72$ & 0,993 & $9,60 \pm 0,55$ & 0,993 \\
\hline & 90 & 4,88 & $53,05 \pm 2,15$ & 0,997 & $12,1 \pm 0,37$ & 0,998 \\
\hline \multirow[t]{5}{*}{22} & 70 & 8,28 & $45,87 \pm 3,49$ & 0,988 & $5,83 \pm 0,51$ & 0,986 \\
\hline & 75 & 8,74 & $54,81 \pm 2,81$ & 0,995 & $6,61 \pm 0,32$ & 0,995 \\
\hline & 80 & 8,64 & $67,82 \pm 2,26$ & 0,998 & $8,48 \pm 0,23$ & 0,999 \\
\hline & 85 & 8,66 & $84,93 \pm 3,30$ & 0,997 & $10,8 \pm 0,32$ & 0,998 \\
\hline & 90 & 8,62 & $100,3 \pm 5,20$ & 0,995 & $13,1 \pm 0,46$ & 0,997 \\
\hline \multirow[t]{5}{*}{32} & 70 & 12,53 & $71,69 \pm 5,29$ & 0,989 & $6,06 \pm 0,46$ & 0,988 \\
\hline & 75 & 13,58 & $92,89 \pm 4,10$ & 0,996 & $7,30 \pm 0,32$ & 0,996 \\
\hline & 80 & 13,14 & $112,6 \pm 4,50$ & 0,997 & $9,28 \pm 0,32$ & 0,997 \\
\hline & 85 & 13,44 & $142,6 \pm 6,48$ & 0,996 & $11,8 \pm 0,41$ & 0,997 \\
\hline & 90 & 13,62 & $172,0 \pm 4,38$ & 0,999 & $14,3 \pm 0,41$ & 0,999 \\
\hline
\end{tabular}

${ }^{a}$ Valores \pm intervalo de confiança a $\mathrm{p}=0,05$.

Tabela 2. Constantes cinéticas de primeira ordem para reações degradativas em purês de pêssego a $75^{\circ} \mathrm{C}$ e para desnaturação térmica da PPO e POD.

\begin{tabular}{|c|c|c|c|}
\hline Reações degradativas & $\begin{array}{c}\mathrm{k} \\
\left(\min ^{-1}\right)\end{array}$ & $\begin{array}{c}\text { Concentração } \\
\text { do purê } \\
\left({ }^{\circ} \text { Brix }\right)\end{array}$ & Fonte \\
\hline do ácido ascórbico & $6,10.10^{-4}$ & 12 & Tabela 3.1 \\
\hline da coloração para 'L' & $3,42.10^{-5}$ & Não & \multirow{3}{*}{$\begin{array}{l}\text { ÁVILA; SILVA } \\
\text { (1999) }\end{array}$} \\
\hline da coloração para 'a' & $3,68.10^{-5}$ & informado & \\
\hline da coloração para 'b' & $4,34 \cdot 10^{-5}$ & & \\
\hline da sacarose & $7,1.10^{-4}$ & 11 & GARZA et al. 1999) \\
\hline da PPO-Granada & 1,4 & - & \multirow{2}{*}{$\begin{array}{l}\text { TORALLES et al. } \\
\text { (2005) }\end{array}$} \\
\hline da POD-Granada & 6,2 & - & \\
\hline
\end{tabular}

córbico também foi maior em altas concentrações (JOHNSON; BRADDOCK; CHEN, 1995).

\subsection{Parâmetros de Arrhenius e $Q_{10}$}

O gráfico de Arrhenius para constante de velocidade de degradação do ácido ascórbico é apresentado na Figura 4. Os dados foram analisados por regressão linear com uma variação explicada em torno da média superior a $99 \%$, e o modelo de Arrhenius foi estatisticamente significativo $(\mathrm{p} \leq 0,01)$ para 12 , 22 e $32^{\circ}$ Brix.

Da inclinação da reta obtida - Ea/R, Figura 4, uma energia de ativação de $46,3 \mathrm{~kJ} \cdot \mathrm{mol}^{-1}$ foi calculada para purê de pêssego a $12{ }^{\circ}$ Brix. Procedimento semelhante foi utilizado para obter $\mathrm{E}_{\mathrm{a}}$ para 22 e $32^{\circ}$ Brix. Os parâmetros de Arrhenius para degradação do ácido ascórbico em purê de pêssego, bem como o coeficiente de reação $Q_{10}$ estão listados na Tabela 3. Os valores de $Q_{10}$ foram maiores entre 80 e $90{ }^{\circ} \mathrm{C}$. A energia de ativação média foi na ordem de $45 \mathrm{~kJ} \cdot \mathrm{mol}^{-1}$ e foi independente da concentração de sólidos solúveis. Para degradação do ácido ascórbico em suco
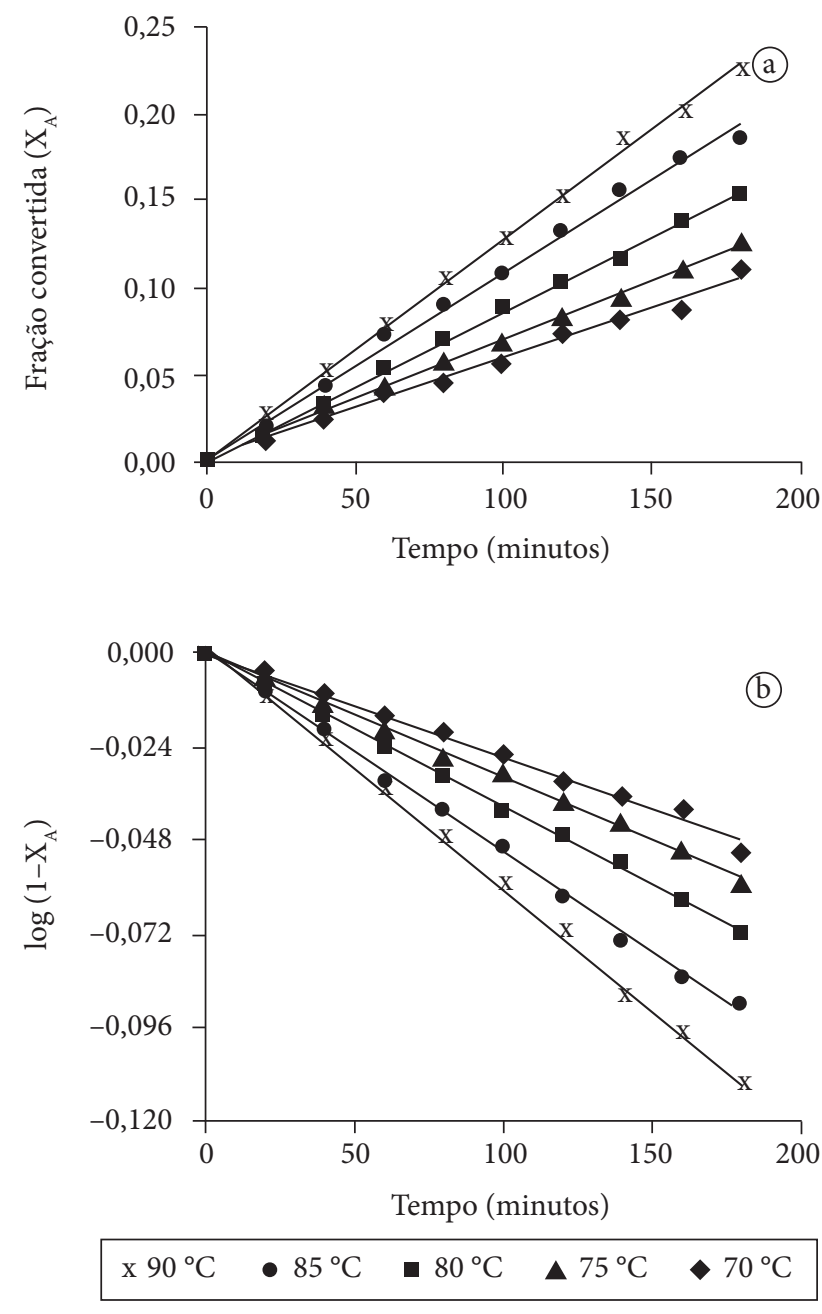

Figura 2. Cinética de degradação do ácido ascórbico em purê de pêssego a $32^{\circ}$ Brix entre 70 e $90^{\circ} \mathrm{C}$ : a) ordem zero; e b) primeira ordem. 
Tabela 3. Parâmetros de Arrhenius e coeficiente de reação $\left(\mathrm{Q}_{10}\right)$ para degradação de ácido ascórbico em purê de pêssego.

\begin{tabular}{|c|c|c|c|c|c|c|}
\hline \multirow{2}{*}{$\begin{array}{c}\text { Concent. } \\
{ }^{\circ} \text { Brix } \\
\end{array}$} & \multicolumn{4}{|c|}{ Parâmetros de Arrhenius } & \multicolumn{2}{|c|}{ Coeficiente de temperatura $\left(\mathrm{Q}_{10}\right)$} \\
\hline & Intervalo $\left({ }^{\circ} \mathrm{C}\right)$ & $\ln \mathrm{A}^{\mathrm{a}}$ & $\mathrm{E}_{\mathrm{a}}^{\mathrm{a}}\left(\mathrm{kJ} \cdot \mathrm{mol}^{-1}\right)$ & $\mathrm{R}^{2}$ & $\left(70-80^{\circ} \mathrm{C}\right)$ & $\left(80-90^{\circ} \mathrm{C}\right)$ \\
\hline 12 & $70-90$ & $8,6 \pm 2,1^{* *}$ & $46,3 \pm 4,5^{* *}$ & 0,997 & 1,51 & 1,61 \\
\hline 22 & $70-90$ & $7,8 \pm 4,0^{\star *}$ & $43,7 \pm 11,7^{\star *}$ & 0,988 & 1,45 & 1,55 \\
\hline 32 & $70-90$ & $8,5 \pm 1,9^{* *}$ & $45,4 \pm 5,5^{\star *}$ & 0,997 & 1,53 & 1,54 \\
\hline
\end{tabular}

Valor \pm intervalo de confiança a $\mathrm{p}=0.05 ; \mathrm{e}^{* *}$ Significativo $(\mathrm{p} \leq 0,01)$.

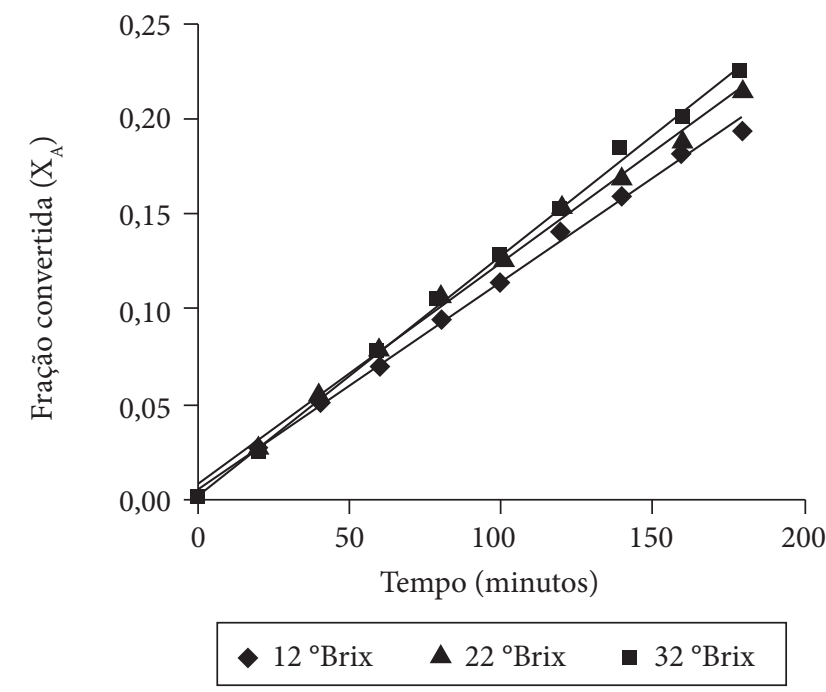

Figura 3. Cinética de degradação do ácido ascórbico com incremento do tempo de aquecimento a $90{ }^{\circ} \mathrm{C}$ para três concentrações de purê de pêssego.

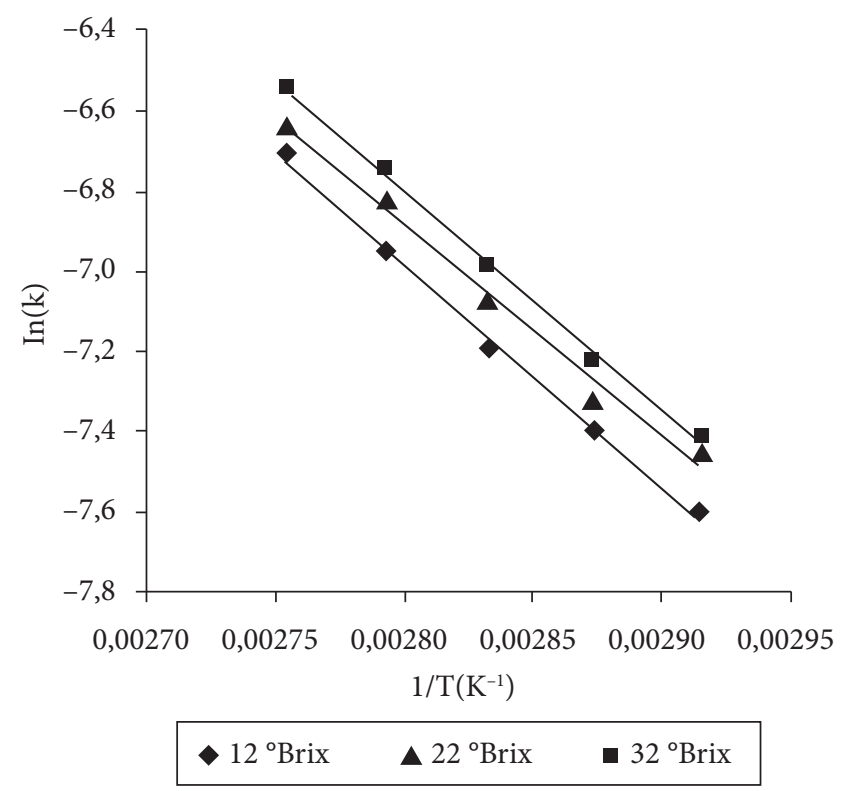

Figura 4. Representação gráfica de Arrhenius da constante cinética de primeira ordem para degradação do ácido ascórbico em purê de pêssego.

concentrado de laranja, Johnson, Braddock e Chen (1995) encontraram uma $\mathrm{E}_{\mathrm{a}}$ média na ordem de $124 \mathrm{~kJ} . \mathrm{mol}^{-1}$. Para degradação da cor do purê de pêssego durante o processamento térmico, uma $\mathrm{E}_{\mathrm{a}}$ média de $108 \mathrm{~kJ} \cdot \mathrm{mol}^{-1}$ foi determinada por
Ávila e Silva (1999). Ambos têm uma cinética mais lenta do que a degradação do ácido ascórbico em purê, o que explica a sua menor $\mathrm{E}_{\mathrm{a}}$.

\section{Conclusões}

A velocidade de degradação do ácido ascórbico foi maior a $90{ }^{\circ} \mathrm{C}$. Em todas as concentrações de purês estudadas, a degradação do ácido ascórbico foi bem descrita usando modelos cinéticos de zero e primeira ordem e, a dependência da temperatura foi significativamente representada pela lei de Arrhenius. A energia de ativação para degradação do ácido ascórbico foi na ordem de $45 \mathrm{~kJ} . \mathrm{mol}^{-1}$ e revelou-se independente da concentração de sólidos solúveis ( $\left.{ }^{\circ} \mathrm{Brix}\right)$. Os conhecidos benefícios da adição do ácido ascórbico no processamento do purê de pêssego foram bem maiores do que as mudanças de cor originárias de sua degradação durante o processamento.

\section{Referências bibliográficas}

AOAC - Official Methods of Analysis of AOAC International/Dr Willian Horwitz, 17. ed. Maryland: AOAC International, 2000.

ASHURST, P. R. Production and Packaging of Non-Carbonated Fruit Juices and Fruit Beverages. 2. ed. London: Blackie Academic, 1995.

ÁVILA, I. M. L. B.; SILVA, C. L. M. Modeling kinetics of thermal degradation of colour in peach puree. Journal of Food Engineering, v. 39, p. 161-166, 1999.

BABSKY, N. E.; TORIBIO, J. L.; LOZANO, J. E. Influence of storage on the composition of clarified apple juice concentrate. Journal of Food Science, v. 51, n. 3, p. 564-567, 1986.

BUERA, M. P. et al. Nonenzymatic browning in liquid model system of hight activity: kinetics of color changes due to caramelization of various single sugars. Journal of Food Science, v. 52, n. 4, p. 1059-1062, 1987.

BUGLIONE, M.; LOZANO, J. Nonenzymatic browning and chemical changes during grape juice storage. Journal of Food Science, v. 67, n. 4, p. 1059-1062, 2002

BURDURLU, H. S.; KARADENIZ, F. Effect of storage on nonenzymatic browning of apple juice concentrates. Food Chemistry, v. 80, p. 91-97, 2003.

FENNEMA, O. R. Química de los alimentos. 2. ed. Zaragoza: Editorial Acribia, 1993.

FREEDMAN, L.; FRANCIS, F. J. Effect of ascorbic acid on color of jellies. Journal of Food Science, v. 49, n. 4, p. 1212-1213, 1984.

GARZA, S. et al. Non-enzimatic in peach pure during heating. Food Research International, v. 32, n. 5, p. 335-343. 1999.

GIRNER, J. et al. Inactivation of peach polyphenoloxidase by exposure to pulsed electric fields. Journal of Food Science, v. 67, n. 4, p. 264-267, 2002. 
HOSNEY, R. C. Chemical changes in carbohydrates procedure by thermal processing. Journal of Chemical Education. v. 61, n. 4, p. 308-312, 1984.

KOCA, N.; SELEN BURDURLU, H.; KARADENIZ, F. Kinetics of nonenzymatic browning reaction in citrus juice concentrates during storage. Turk Journal Agricultural, v. 27, p. 353-360, 2003.

JOHNSON, J. R.; BRADDOCK, R. J.; CHEN, C. S. Kinetics of ascorbic acid loss and nonenzymatic browning in orange juice serum: experimental rate constants. Journal of Food Engineering, v. 60, n. 3, p. 502-505, 1995.

LEE, H. S.; NAGY, S. Quality changes nonenzymatic browning intermediates in grapefruit juice during storage. Journal of Food Science, v. 53, n. 1, p. 168-172, 1988.

LOZANO, J. E.; IBARZ, A. Colour changes in concentrated fruit pulp during heating at high temperatures. Journal of Food Engineering, v. 31, n. 3, p. 365-373, 1997.

LUH, B. S. Nectars, pulpy juice and fruit juice blends. In: Fruit and vegetable juice processing technology. Nelson, $\mathrm{P}$ and Tressler, D. ed., Wesport: AVI, 1980. cap. 10, p. 436-505.

MARTÍ, N.; PÉREZ-VICENTE, A.; GARCIA-VIGUERA, C. Influence of storage temperature and ascorbic acid addition on pomegranate juice. Journal of the Science of Food and Agriculture, v. 82, n. 2, p. 217-221, 2001.

OKTAY, M. et al. Polyphenoloxidase from Amaya apple. Journal of Food Science. v. 60, n. 3, p. 494-496, 1995.

OZKAN, M.; KIRCA, A.; CEMEROGLU, B. Effects of hydrogen peroxide on the stability of ascorbic acid during storage in various fruit juices. Food Chemistry, v. 88, p. 591-597, 2004.

PETRIELLA, C. et al. Kinetics of deteriorative reactions in model food systems of high water activity: color changes due to nonenzymatic browning. Journal of Food Science. v. 50, n. 3, p. 622-626, 1985.
PORRETTA, S. Nonenzymatic browning of tomato products. Food Chemistry, v. 40, n. 3, p. 323-335, 1991.

ROBERTSON, L. M.; SAMANIEGO, H. T. Effect of initial dissolved oxygen levels on the degradation of ascorbic acid and the browning of lemon juice during storage. Journal of Food Science. v. 51, n. 1, p. 184-187, 192, 1986.

SMOOT, J. M.; NAGY, S. Effect of storage temperature and duration on total vitamina $\mathrm{C}$ content of canned single-strength grapefruit juice. Journal Agricultural and Food Chemistry, v. 28, p. 417-421, 1980.

TORALLES, R. T. et al. Partial characterization of the enzymatic browning for polyphenoloxidase in peaches of the cv Ganada, Jade, Esmeralda and Maciel. Revista Brasileira de Agrociência, v. 10, n. 2, p. 241-244, 2004a.

TORALLES, R. P. et al. Inativação da polifenoloxidase pela interação de calor, $\mathrm{pH}$ e ácido ascórbico em pêssego da Cv. Granada usando MSR. In: XIX CBCTA - Ciência e Tecnologia de Alimentos: Estratégia para o Desenvolvimento, 2004, Recife. Anais... Recife: CBCTA. 2004b.

TORALLES, R. P. et al. Properties of polyphenoloxidase and peroxidase from Granada clingstone peaches. Brazilian Journal of Food Technology, v. 8, n. 3, p. 233-242, 2005.

VÁMOS-VIGIÁZÓ, L. Polyphenol oxidase and peroxidase in fruits and vegetables. CRC - Critical Reviews in Food Science and Nutrition, v. 15 , n. 1, p. 49-127, 1981.

WHITAKER, J. R. Principles of enzimology for the food sciences. 2. ed. New York: Marcel Dekker, 1994. p. 625.

WONG, M.; STANTON, D. W. Effect of removal of amino acids and phenolic compounds on non-enzymatic browning in stored kiwifruit juice concentrates. Lebensmittel-WissenschaftundTechnologie, v. 26, n. 2, p. 138-144, 1993. 